\title{
Correlation between self-esteem and stress response in Chinese college students: The mediating role of the need for social approval
}

\author{
Juan Yang a,b,*,1, Yu Yang ${ }^{\mathrm{a}, \mathrm{b}, 1}$, Haijiang $\mathrm{Li}^{\mathrm{a}}{ }^{\mathrm{a}, \mathrm{b}}$, Yan Hou ${ }^{\mathrm{a}, \mathrm{b}}$, Mingming Qi ${ }^{\mathrm{a}, \mathrm{b}}$, Lili Guan ${ }^{\mathrm{a}, \mathrm{b}}$, Xianwei Che ${ }^{\mathrm{a}, \mathrm{b}}$, \\ Yu Chen ${ }^{\mathrm{a}, \mathrm{b}}$, Weihai Chen ${ }^{\mathrm{a}, \mathrm{b}}$, Jens C. Pruessner ${ }^{\mathrm{c}}$ \\ ${ }^{a}$ Key Laboratory of Cognition and Personality, Southwest University, Chongqing 400715, China \\ ${ }^{\mathrm{b}}$ Department of Psychology, Southwest University, Chongqing 400715, China \\ ${ }^{\mathrm{c}}$ Douglas Hospital Research Center, McGill University, Montreal, Quebec, Canada
}

Keywords:

Self-esteem

Stress

Salivary cortisol

The need for social approval

Internal locus of control

Mediation analysis

\begin{abstract}
A B S T R A C T
Interpersonal theories of self-esteem assume that the importance that others place on oneself contributes to individual levels of self-esteem. Recent studies further suggest a possible link between self-esteem and the endocrine stress response, mediated through individual levels of locus of control, without taking levels of social approval into account. The present set of studies aimed to explore the correlation between self-esteem and stress response in Chinese students, and simultaneously take into account the possible mediating role of internal locus of control and need for social approval. In study one, twenty-eight college students' heart rates and saliva samples were collected while they underwent the Trier Social Stress Test (TSST). Their self-esteem scores were assessed using the Rosenberg self-esteem scale. Results showed a positive correlation between self-esteem and their endocrine stress responses to the TSST. In study two, forty-one college students' heart rates were collected while they underwent the TSST. Their locus of control scores were assessed by the Internality, Powerful Others, and Chance Scale, and their social approval scores were assessed by the Marlowe-Crowne Social Desirability Scale. Results indicated a significant positive correlation between self-esteem and heart rate changes during the TSST, with a mediating role of the need for social approval in the association between self-esteem and heart rate stress responses. We speculate that cultural differences may moderate the association between self-esteem and stress response.
\end{abstract}

\section{Introduction}

Previous psychoneuroendocrinological studies have consistently reported a role of self-esteem in the perception and processing of stress (Kirschbaum et al., 1995; Pruessner, Hellhammer, \& Kirschbaum, 1999; Pruessner et al., 2005). In one such study, the association between self-esteem and free cortisol stress response with regard to experimentally induced success or failure was investigated. Results showed a significant negative correlation between the free cortisol response to this stress task and selfesteem in the failure condition (Pruessner et al., 1999). In another study, 20 healthy male subjects were exposed five times to an identical stressor (the Trier social stress test; TSST), a task designed to induce moderate psychosocial stress in a laboratory setting

\footnotetext{
* Corresponding author at: Department of Psychology, Southwest University, Chongqing 400715, China. Tel.: +86 236836 7697, +86 2368253629.

E-mail address: valleyqq@swu.edu.cn (J. Yang).

${ }^{1}$ Co-first author.
}

through a combination of public speaking and mental arithmetic. Interestingly, about one third of the tested population displayed large increases to each of the five experimental treatments instead of habituating to the repeated stress exposure. Psychometric analysis suggested that low self-esteem was the best predictor of a lack of habituation of the cortisol response to repeated stress exposure (Kirschbaum et al., 1995). In another study with young subjects, low self-esteem again predicted a higher cortisol stress response when subjects were exposed to the Montreal Imaging Stress Task (MIST), a combination of social evaluative and mentally challenging tasks (Pruessner et al., 2005). Low self-esteem has further been shown to be associated with greater cortisol reactivity in a rejection stress task (Ford \& Collins, 2010).

One possible biological mechanism underlying this association involves appraisal theories. Since self-esteem and internal locus of control are usually highly correlated, it has been postulated that in the evaluation of whether a given situation is perceived as stressful, self-esteem and internal locus of control systematically interact with situational factors (Pruessner, 2009). Low self-esteem 
individuals attribute little importance to oneself, and speculate that they have little impact on the outcome of their actions, thus finding more situations uncontrollable and unpredictable, and consequently will be evaluating more situations as stressful. Conversely, high self-esteem individuals tends to be strongly associated with internal locus of control, or the confident perception that one's outcomes are determined by one's actions; therefore, they would not interpret the same situations as stressful as low self-esteem individuals do (Pruessner, 2009).

Although traditional intrapersonal theorists have conceptualized self-esteem as a person's private self-evaluation of one's goodness or worth (MacDonald, Saltzman, \& leary, 2003), interpersonal theories of self-esteem suggest that people's thoughts and feelings about themselves reflect, in part, how they believe they are perceived and evaluated by others (Leary, Haupt, Strausser, \& Chokel, 1998; Leary, Tambor, Terdal, \& Downs, 1995). For example, according to sociometer theory, others' reactions have such a strong effect on self-esteem because the self-esteem system itself is a subjective monitor or gauge of the degree to which the individual is being accepted and rejected by other people (Leary, 1999; Leary \& Baumeister, 2000).

Another factor that would affect the form and sources of self-esteem is cultural difference (Luk \& Bond, 1992). Over the past decades, it has been suggested that the construct of individualismcollectivism may be reflected in self-esteem (Tafarodi \& Swann, 1996). Basis of self-esteem in the individualistic culture is the ability to express the self and to validate internal attributes, whereas in the collectivistic culture it is the ability to adjust, to restrain the self, and to maintain harmony with social context (Markus \& Kitayama, 1991). Self-esteem for those in individualistic cultures depends on one's abilities, attributes, and achievements, whereas for those in collectivistic culture, it may be the more public aspects of the self, like one's significant social roles, status, and important interpersonal relations, that would be focal in self-esteem maintenance (Markus \& Kitayama, 1991).

With regard to the possible link between self-esteem and the endocrine stress response, previous studies focused on individual levels of locus of control, however, without taking levels of social approval into account. In the current manuscript, the standardized TSST was used to examine the correlation between self-esteem and stress response in Chinese students in one study, and to examine the mediating role for either internal locus of control and/or need for social approval simultaneously in a second study. On one hand, people's self-esteem largely depends on one's social roles in collectivistic culture and in Chinese context, people are seeing oneself as part of an encompassing social relationship and they are recognizing that one's behavior is determined and to a large extent organized by the thoughts, feelings, and actions of others in the relationship (Markus \& Kitayama, 1991); on the other hand, social evaluative threat is prominent throughout the TSST (Dickerson \& Kemeny, 2004). Therefore, we hypothesized that self-esteem and the need for social approval would interact with situational factors in the evaluation of whether a given situation is perceived as stressful. In a collectivistic culture, specifically in the Chinese context in the current study, high self-esteem individuals attribute a lot of importance to social approval, thus we speculated that they would focus on social desirability when regarding outcome of their actions. Thus, we hypothesized that these individuals would evaluate situations more stressful when they find that the situation is a threat to their needs of social approval. Conversely, low selfesteem tends to be weakly associated with social approval, or the confident perception that one's outcomes are determined by one's evaluation; therefore, we speculated that low self-esteem individuals would not interpret the psychosocial stressor to be as stressful as high self-esteem individuals.

\section{Study 1}

\subsection{Methods}

\subsubsection{Participants}

Thirty healthy University students were recruited by posting flyers at local university buildings and through an advertisement on a website. Upon initial contact, a telephone interview was conducted to establish eligibility for the study through assessment of medical history, and obtain demographic information in case of recruitment. Exclusion criteria consisted of past or current depression, current infections as well as current medication treatment. Two participants were excluded because of their high scores on the Beck Depression Inventory (Beck, 1967), which resulted in a final dataset of 28 students ( 15 male, mean age $21.04 \pm 1.43$ ). All women were free cycling, and were tested during their follicular phase of their menstrual cycle (confirmed by oral reports). All participants refrained from smoking, eating, drinking alcoholic beverages, and physical exercise at least $1 \mathrm{~h}$ prior to testing. After participants were given a complete explanation of the study, written informed consent was obtained. Participants were paid for their participation.

\subsubsection{Measures}

2.1.2.1. Questionnaire. Participants completed the Rosenberg selfesteem scale (RSE), a questionnaire that assesses a person's overall evaluation of his or her self-worth (Rosenberg, 1965). The RSE is made up of 10 items such as 'On the whole, I am satisfied with myself' or 'I feel I do not have much to be proud of' and is coded on a 4-point scale ranging from 1 (strongly disagree) to 4 (strongly agree), with the negative items needing to be reverse scored. Cronbach's $\alpha$ of this scale for the present sample was 0.769 .

2.1.2.2. Endocrine measurement. Saliva samples were taken via salivette sampling device (SARSTEDT, Germany) throughout the experiment in order to assess levels of cortisol. These samples included two samples before the preparation period (baseline, pre-preparation), one before the speech and arithmetic task (preTSST), and six following the TSST (post-TSST, 10, 20, 30, 40, $50 \mathrm{~min}$ ). All cortisol samples were stored in a $-20^{\circ} \mathrm{C}$ freezer until subsequent analyses. Cortisol measures were established by using a time-resolved fluorescence immunoassay. Intra- and inter- assay variability were less than $10 \%$ and $12 \%$, respectively.

2.1.2.3. Heart rate measurement. Heart rate was monitored continuously employing a wireless signal transmission device (Spirit, Netherlands). The sensor is placed on the middle finger of participants' non-dominant hand. Heart rates were averaged across $1 \mathrm{~min}$ intervals.

\subsubsection{Procedure}

Participants were exposed to the TSST between 14:30 and 17:00. During the TSST, participants had to give a speech and performed serial subtractions in front of a committee, usually for a total of about $10 \mathrm{~min}$ (Kirschbaum, Pirke, \& Hellhammer, 1993). The committee consisted of two persons of the opposite sex, who maintained a neutral facial expression throughout the subject's presentation. During the speech, participants were asked to give a mock job interview and to convince the committee that they were the perfect applicant for the vacant position. During the serial subtraction, participants were asked to serially subtract the number 13 from 13,497 as fast and as accurately as possible. Upon committing an error, participants had to restart at 13,497. 


\subsubsection{Data analysis}

One way repeated measures analysis of variance (ANOVA) were conducted separately to examine possible time effects of salivary cortisol and heart rate. Self-esteem and their salivary cortisol level were correlated using Pearson's correlation coefficient. Self-esteem and heart rate stress responses were also correlated using Pearson's correlation coefficient.

\subsection{Results}

Figure 1 summarizes endocrine and cardiovascular stress responses to the TSST. For salivary cortisol, one way repeated ANOVA revealed a significant effect of time, $F(8,216)=5.693$, $p<0.01$. Cortisol in saliva reached its peak 20 min post TSST, $p<0.01$. For heart rate, one way repeated ANOVA also revealed a significant effect of time, $F(8,216)=19.33, p<0.01$, mean heart rate reached its peak under TSST stimulation immediately, $p<0.01$ and dropped to baseline shortly after cessation of the stressor. Further, significant positive correlations were found both between self-esteem and the total salivary cortisol response (measured as area under the curve with respect to ground), $r=0.324$, $p=0.09$ and between self-esteem and the peak heart rate at the onset of the TSST protocol, $r=0.456, p<0.05$ (Fig. 2).

\subsection{Discussion}

Two main findings emerged from the first study. First, the TSST induced significant increases in cortisol and heart rate stress response. This result was consistent with numerous research groups using the TSST to induce a psychological stress responses. It was also consistent with a recent testing result using the same protocol among Chinese adolescents (Fu, Weng, \& Tao, 2012).

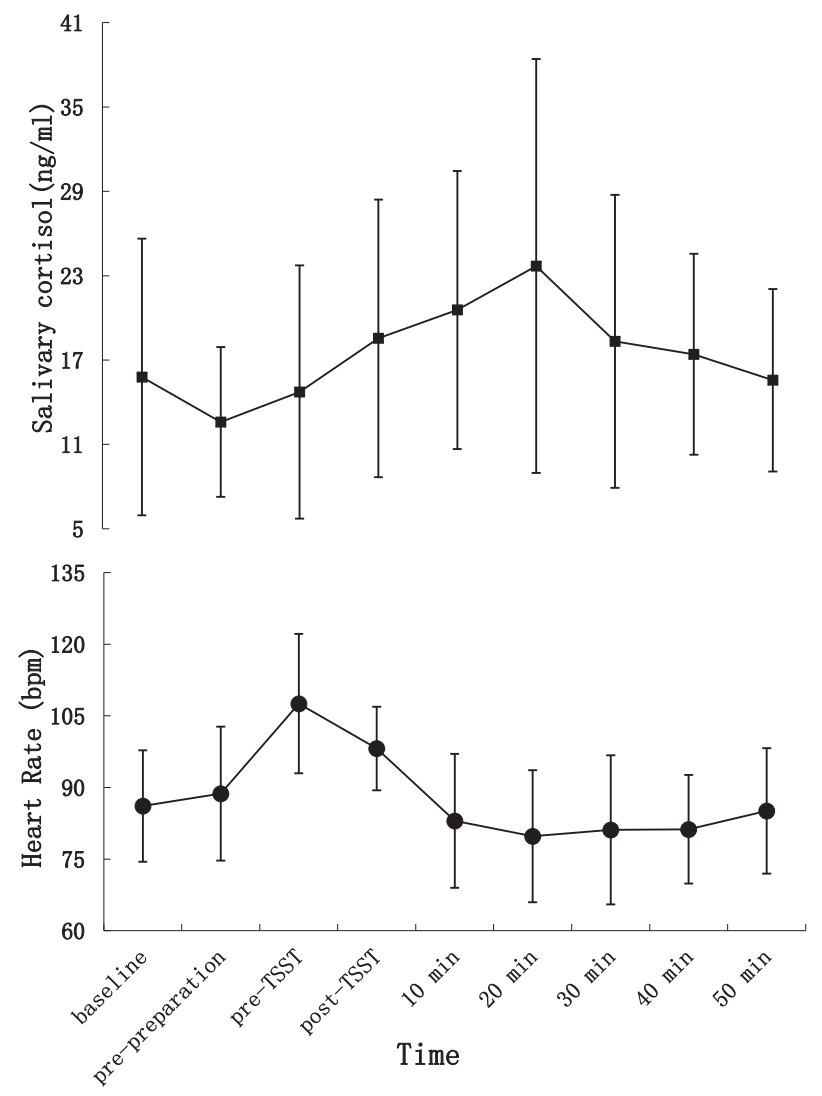

Fig. 1. Salivary cortisol level and heart rate changes in study 1 . Means of salivary cortisol levels and heart rate during the stress and the rest condition. Standard Deviation represented with error bars.
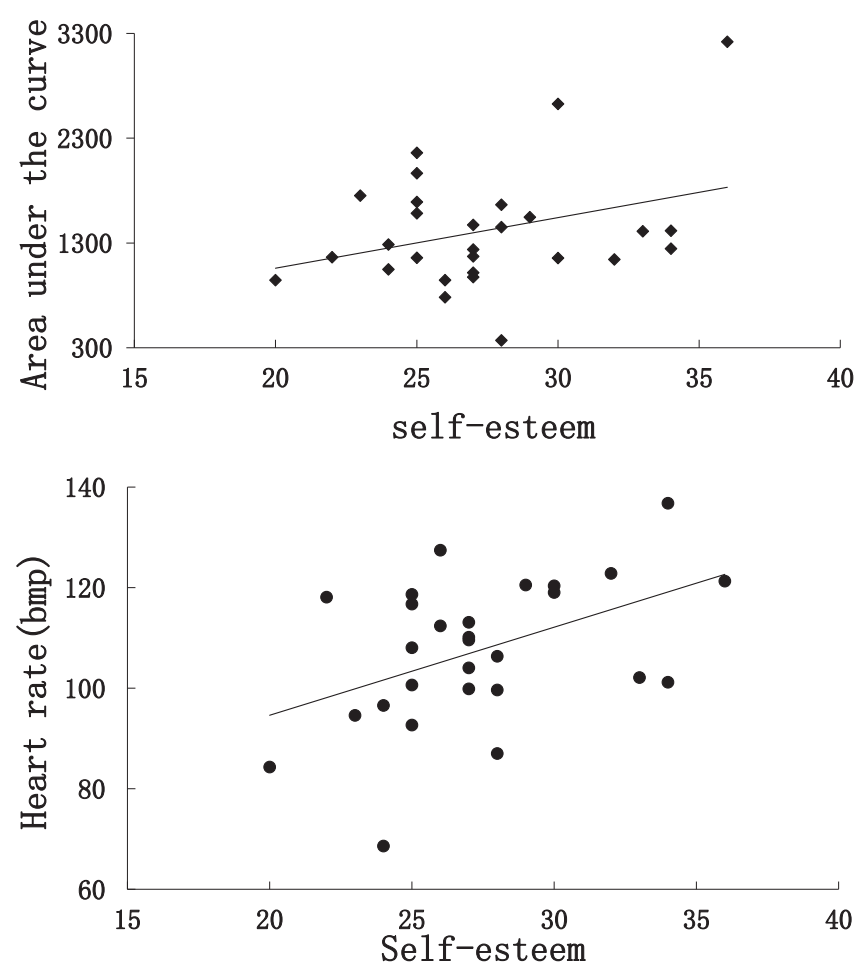

Fig. 2. Correlations between self-esteem and heart rate at the onset of the TSST protocol (upper) and correlations between self-esteem and the total salivary cortisol response (measured as area under the curve with respect to ground) (lower).

Second, a positive correlation was found between self-esteem and stress responses in Chinese, which was consistent with our hypotheses that high self-esteem individuals in collectivistic culture would evaluate the situations as stressful when they found that the situation was a threat to their needs of social approval. In order to further study the mechanisms underlying the association, we proposed and tested a mediational model (see Fig. 3) to explain the links between self-esteem and neuroendocrine responses to stress. According to this model, individuals with high self-esteem would be more likely to appraise the TSST as threatening to their needs of social approval, which would then result in increased stress response (solid lines). Since people's self-esteem would largely depend on one's social roles, other than their

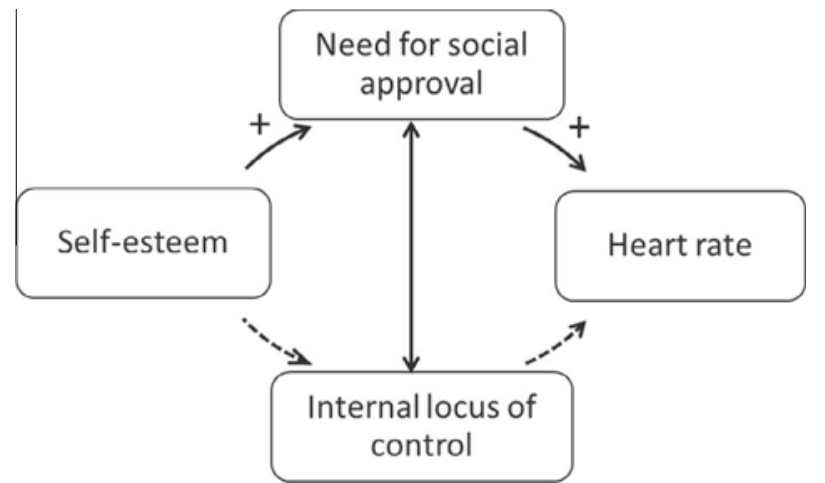

Fig. 3. Hypothetical mediation model of the need for social approval and internal locus of control in the association of self-esteem and stress response. According to this model, individuals with high self-esteem would be more likely to appraise the TSST as threatening to their needs of social approval, which would then result in increased stress response (solid lines). Since people's self-esteem would largely depend on one's social roles, other than their internal attributes in collectivist culture, therefore, their locus of control would not mediate the correlation between self-esteem and stress response in collectivistic culture (dashed lines). 
internal attributes in collectivist culture, therefore, their locus of control would not mediate the correlation between self-esteem and stress response in collectivistic culture (dashed lines).

\section{Study 2}

\subsection{Methods}

\subsubsection{Participants}

Forty-three healthy University students were recruited for the second experiment. Two participants were excluded because of high scores on Beck Depression Inventory, which resulted in a final dataset of forty-one (19 male, 21 female, mean age $=19.78 \pm 1.20$ ). Women were not taking any oral contraceptives and were tested during the follicular phase of their menstrual cycle. All participants refrained from smoking, eating, drinking, alcoholic beverages, and physical exercise at least $1 \mathrm{~h}$ prior to testing. Written informed consent was obtained prior to the testing. Participants were paid for their participation.

\subsubsection{Measures}

3.1.2.1. Questionnaires. Participants completed the Rosenberg selfesteem scale (RSE), a questionnaire that assesses a person's overall evaluation of his or her self-worth (Rosenberg, 1965). The RSE is made up of 10 items such as 'On the whole, I am satisfied with myself' or 'I feel I do not have much to be proud of' and is coded on a 4-point scale ranging from 1 (strongly disagree) to 4 (strongly agree), with the negative items needing to be reverse scored. Cronbach's $\alpha$ of this scale for the present sample was 0.813 .

Locus of control was measured using the Levenson I, P, and C Scale (Levenson, 1981). The I, P, and C Scales yield scores for each of the dimensions of locus of control. Each scale contains eight items and is presented in Likert scale format. The I scale measures internality or the degree to which a person believes that he or she is in control of or responsible for events occurring in his or her own life, and the P and C scales measure externality. The P scale measures the degree to which a person believes that other people (powerful others) are in control of his or her life and the $C$ scale measures the degree to which a person believes that chance or luck is in control of his or her life. Cronbach's $\alpha$ of this scale for the present sample was 0.614 . Since we focused on participants' internal locus of control, only the scores of I scale were used.

The Marlowe-Crowne Social Desirability Scale (MCSDS) is a 33item self-report questionnaire, which uses a forced choice, TrueFalse format for responding to items (Crowne \& Marlowe, 1960). Total scores range from zero (low) to 33 (high) on a social desirability scale. The MCSDS has two factors: Attribution and Denial. Eighteen items make up the Attribution subscale, which addresses an individual's propensity to endorse items depicting socially approved, but uncommon behaviors. Fifteen items make up the Denial subscale, which addresses the tendency to deny socially disapproved, but common behaviors. Cronbach's $\alpha$ of this scale for the present sample was 0.742 .

3.1.2.2. Heart rate measurement. Heart rate was monitored continuously employing a wireless signal transmission device (Spirit, Netherlands). Mean heart rate responses were available for a baseline period, the task preparation period, as well as the task period, $30 \mathrm{~min}$ and $40 \mathrm{~min}$ post-stress period. The heart rate value during the task period was averaged across $1 \mathrm{~min}$; all others were averaged across $10 \mathrm{~min}$.

\subsubsection{Procedure}

Participants finished questionnaires first and then they were exposed to the TSST between 15:30 and 17:30. The order of the questionnaires were counterbalanced between participants. The protocol of the TSST was identical to that of the first study.

\subsubsection{Data analysis}

One way repeated measures analysis of variance was conducted to examine the possible time effect of heart rate. Based on Fig. 3, path analyses via structural equation model using Amos 5.0 were conducted to test the mediation effect of the need for social approval and/or locus of control in the relationship between selfesteem and heart rate response.

\subsection{Results}

Figure 4 shows the heart rate stress responses to the TSST. One way repeated ANOVA revealed a significant effect of time, $F$ $(4,160)=82.19, p<0.01$, with mean heart rate changes peak during TSST, $p<0.001$. The means and standard deviations for selfesteem, internal locus of control, need for social approval and heart rates during the task are given in Table 1, along with the correlation coefficients between these variables. Results showed that self-esteem was significantly correlated with internal locus of control, need for social approval and heart rate stress response. Further, heart rate stress response was positively correlated with the need for social approval.

To test the mediation model, path analyses were conducted according to Fig. 3. After deleting non-significant paths, the final model shown in Fig. 5 was obtained. The model fitted the data well, $d f=2, \quad \chi^{2}=1.988 \quad(p=0.37), \quad \mathrm{NFI}=0.934, \quad \mathrm{CFI}=1.00, \quad \mathrm{IFI}=1.00$, RMSEA $=0.00$. Furthermore, bootstrapping was conducted to test the mediating effects shown in Fig. 5. The 95\% bias-corrected bootstrapping confidence intervals (CIs) with 2000 re-samples of the indirect effects of self-esteem on heart rate stress response through need for social approval was 0.0064-0.132, suggesting that the mediating effect of need for social approval was significant. However, the mediating effect of internal locus of control was not supported.

\subsection{Discussion}

Study 2 took a further step exploring the mechanism underlying the association between self-esteem and the stress response. Firstly, a significant positive correlation between self-esteem and heart rate stress response was observed, which was consistent with the results of study 1 . Secondly, data of the present study supported the mediating effect of the need for social approval in the association between self-esteem and heart rate stress response. Although self-esteem was indeed positively correlated with internal locus of control, path analyses did not support the mediating effects of internal locus of control.

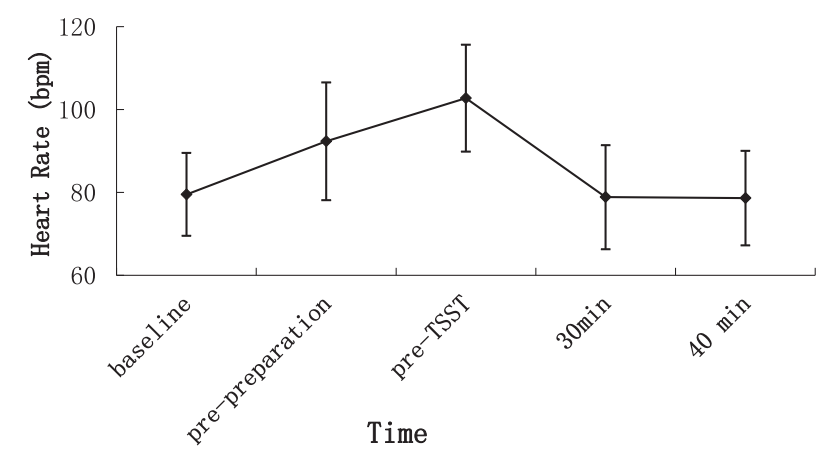

Fig. 4. Heart rate changes during the stress and the rest condition in study 2 . Standard Deviation represented with error bars. 
Table 1

Descriptive statistics and correlations $(N=41)$

\begin{tabular}{lrrllll}
\hline Variable & \multicolumn{1}{c}{$M$} & \multicolumn{1}{c}{ SD } & \multicolumn{1}{c}{1} & \multicolumn{1}{l}{2} & 3 & 4 \\
\hline 1. Self-esteem & 28.7 & 3.7 & & & & \\
2. Internal locus of control & 31.3 & 4.3 & $0.407^{* * *}$ & & & \\
3. Need for social approval & 15.6 & 5.2 & $0.566^{* * *}$ & 0.095 & & \\
4. Heart rate & 102.7 & 12.9 & $0.335^{*}$ & 0.19 & $0.315^{*}$ &
\end{tabular}

${ }^{*} p<0.05$.

$p<0.01$.

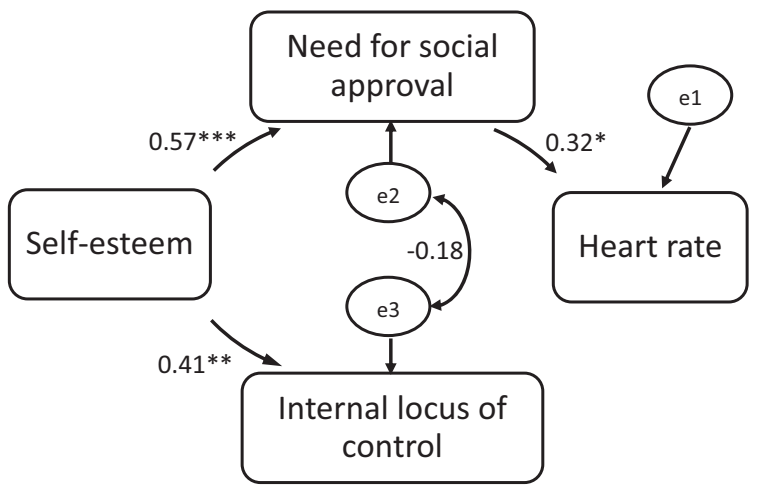

Fig. 5. Path analyses of the mediation model of self-esteem, the need for socia approval, internal locus of control and heart rate in study 2 . The correlation between error terms describe the correlation between the need for social approval and the internal locus of control. ${ }^{*} p<.05,{ }^{* *} p<.01,{ }^{* * *} p<.001$.

\subsection{General discussion}

The present study aimed to explore the correlation between self-esteem and stress response in Chinese students, while simultaneously taking into account the possible mediating role of internal locus of control and/or need for social approval. Results suggest that subject's self-esteem and their endocrine stress responses were positively correlated when they underwent the TSST. It further indicated a mediating role of the need for social approval in the association between self-esteem and heart rate stress response.

Both study one and study two found that participants' selfesteem was positively correlated with their objective stress response (e.g., heart rate and salivary cortisol) in a population of Chinese students. Although both studies have used the same TSST protocol as the stress stimulation, different participants at different times in the two studies were tested, thus replicating the positive correlation. However, this positive correlation was inconsistent with previous studies showing an inverse association between self-esteem and the neuroendocrinological stress response (Kirschbaum et al., 1995; Pruessner et al., 2005). Since both the present studies and the previous studies collected participants' heart rate and/or salivary cortisol, the contrary results between studies were likely not caused by differences in stress indicators.

To further explore the inconsistency and to explain the mechanisms between self-esteem and neuroendocrine responses to stress, the mediation role of internal locus of control and/or the need for social approval were tested in study two. Results supported the mediating effect of the need for social approval in Chinese participants. However, the possible mediation effect of locus of control was not supported in the present study. We cautiously speculated that cultural differences might moderate the association between self-esteem and stress response.

Self-esteem for those in individualistic cultures depends on one's abilities, attributes, and achievements (Luk \& Bond, 1992;
Markus \& Kitayama, 1991), and it has been postulated that in the evaluation of whether a given situation is perceived as stressful, self-esteem and internal locus of control systematically interact with situational factor (Pruessner, 2009). High self-esteem tends to be strongly associated with internal locus of control, or the confident perception that one's outcomes are determined by one's actions; therefore, they would not interpret the same situations as stressful as low self-esteem individuals do (Pruessner, 2009). However, in a collectivistic culture, people's self-esteem largely depends on one's social roles (Markus \& Kitayama, 1991), and it could be suggested that self-esteem and the need for social approval might interact with situational factors in the evaluation of whether a given situation is perceived as stressful. High selfesteem individuals attribute a lot importance to social approval, and speculate that they could potentially gain a lot of social desirability depending on the outcome of their actions, thus evaluating situations more stressful when they find that the situation is a threat to their needs of social approval. This could likely explain the contradictory findings between this and earlier studies, and would put the focus on cultural factors.

Some limitations of this study must be noted. First, sample sizes were small and [some] results were only marginally significant; this limits the conclusions that can be drawn from the current set of studies. Second, although the present study speculated that cultural differences may moderate the association between selfesteem and stress response, a more direct confirmation would include collecting data from an individualistic culture in the same experiment. Future studies should explore the possible cultural differences between self-esteem and the stress response, and further test the possible mediating role for either internal locus of control or need for social approval both in westerner culture and in eastern culture.

Some suggestions for future work in this area are also provided. First, the biopsychosocial model of challenge and threat suggests to differentiate between challenge and threat in stressful situations, and it also calls for the assessment for cardiovascular responses as indexes of challenge and threat motivational states (Seery, Blascovich, Weisbuch, \& Vick, 2004). Future studies may explore this model to differentiate the challenge and threat motivational states in participants. Second, researchers have suggested that a sole focus on self-esteem level and neglecting self-esteem instability is likely to give an incomplete picture on the relationship between stress and physiological reactivity (Kernis, Cornell, Sun, Berry, \& Harlow, 1993). Thus, future studies should assess selfesteem stability along with self-esteem level as well.

\section{Acknowledgement}

This work was supported by the Fundamental Research Funds for the Central Universities (SWU1309105, SWU1009092).

\section{References}

Beck, A. T. (1967). Depression: Clinical, experimental, and theoretical aspects. New York: Harper \& Row.

Crowne, D. P., \& Marlowe, D. (1960). A new scale of social desirability independent of psychopathology. Journal of Consulting Psychology, 24(4), 349-354.

Dickerson, S. S., \& Kemeny, M. E. (2004). Acute stressors and cortisol response: A theoretical integration and synthesis of laboratory research. Psychological Bulletin, 130, 355-391.

Ford, M. B., \& Collins, N. L. (2010). Self-esteem moderates neuroendocrine and psychological responses to interpersonal rejection. Journal of Personality and Social Psychology, 98(3), 405-419.

Fu, J., Weng, T., \& Tao, F. (2012). Cortisol response to the Trier Social Stress test among Chinese adolescents. Journal of Pediatric Endocrinology and Metabolism, 25(11-12), 1213-1216.

Kernis, M. H., Cornell, D. P., Sun, C. R., Berry, A., \& Harlow, T. (1993). There's more to self-esteem than whether it is high or low: The importance of stability of selfesteem. Journal of Personality and Social Psychology, 65(6), 1190-1204. 
Kirschbaum, C., Pirke, K. M., \& Hellhammer, D. H. (1993). The 'Trier Social Stress Test' - A tool for investigating psychobiological stress responses in a laboratory setting. Neuropsychobiology, 28(1-2), 76-81.

Kirschbaum, C., Prussner, J. C., Stone, A. A., Federenko, I., Gaab, J., Lintz, D., et al. (1995). Persistent high cortisol responses to repeated psychological stress in a subpopulation of healthy men. Psychosomatic Medicine, 57(5), 468-474.

Leary, M. R. (1999). Making sense of self-esteem. Current Directions in Psychological Science, 8(1), 32-35.

Leary, M. R., \& Baumeister, R. F. (2000). The nature and function of self-esteem: Sociometer theory. Advances in Experimental Social Psychology, 32, 1-62.

Leary, M. R., Haupt, A. L., Strausser, K. S., \& Chokel, J. T. (1998). Calibrating the sociometer: The relationship between interpersonal appraisals and state selfesteem. Journal of Personality and Social Psychology, 74(5), 1290-1299.

Leary, M. R., Tambor, E. S., Terdal, S. K., \& Downs, D. L. (1995). Self-esteem as an interpersonal monitor: The sociometer hypothesis. Journal of Personality and Social Psychology, 68(3), 518-530.

Levenson, H. (1981). Differentiating among internality, powerful others, and chance. In H. M. Lefcourt (Ed.), Research with the locus of control construct. New York: Academic Press.

Luk, C. L., \& Bond, M. H. (1992). Explaining Chinese self-esteem in terms of the selfconcept. Psychologia, 35, 147-154.
MacDonald, G., Saltzman, G. L., \& leary, M. R. (2003). Social approval and trait selfesteem. Journal of Research in Personality, 37, 23-40.

Markus, H. R., \& Kitayama, S. (1991). Culture and the self: Implications for cognition, emotion, and motivation. Psychological Review, 98(2), 224-253.

Pruessner, J. C., Baldwin, M. W., Dedovic, K., Renwick, R., Mahani, N. K., Lord, C., et al. (2005). Self-esteem, locus of control, hippocampal volume, and cortisol regulation in young and old adulthood. Neuroimage, 28(4), 815-826.

Pruessner, J. C., Hellhammer, D. H., \& Kirschbaum, C. (1999). Low self-esteem, induced failure and the adrenocortical stress response. Personality and individual differences, 27, 477-489.

Pruessner, J. C. (2009). Regulation of the stress-response: Self-esteem and the stress response. In B. S. McEwen (Ed.), New encyclopedia of neuroscience (pp. 489-493). Oxford, UK: Elsevier.

Rosenberg, M. (1965). Society and the adolescent self-image. Princeton, NJ: Princeton University Press.

Seery, M. D., Blascovich, J., Weisbuch, M., \& Vick, S. B. (2004). The relationship between self-esteem level, self-esteem stability, and cardiovascular reactions to performance feedback. Journal of Personality and Social Psychology, 87(1), $133-145$.

Tafarodi, R. W., \& Swann, W. B. (1996). Individualism-collectivism and global selfesteem: Evidence for a cultural trade-off. Journal of Cross-Cultural Psychology, 27(6), 651-672. 